\title{
Strange nuclear matter within Brueckner-Hartree-Fock theory
}

\author{
I. Vidaña, A. Polls, and A. Ramos \\ Departament d'Estructura i Constituents de la Matèria, Universitat de Barcelona, E-08028 Barcelona, Spain \\ M. Hjorth-Jensen \\ Department of Physics, University of Oslo, N-0316 Oslo, Norway \\ V. G. J. Stoks \\ Centre for the Subatomic Structure of Matter, University of Adelaide, Adelaide SA 5005, Australia
}

(Received 10 September 1999; published 20 January 2000)

\begin{abstract}
We have developed a formalism for microscopic Brueckner-type calculations of dense nuclear matter that includes all types of baryon-baryon interactions and allows us to treat any asymmetry in the fractions of the different species $\left(n, p, \Lambda, \Sigma^{-}, \Sigma^{0}, \Sigma^{+}, \Xi^{-}\right.$, and $\left.\Xi^{0}\right)$. We present results for the different single-particle potentials, focusing on situations that can be relevant in future microscopic studies of beta-stable neutron star matter with strangeness. We find that both the hyperon-nucleon and hyperon-hyperon interactions play a non-negligible role in determining the chemical potentials of the different species.

PACS number(s): 26.60.+c, 21.65.+f, 13.75.Ev, 21.30.-x
\end{abstract}

\section{INTRODUCTION}

The properties and composition of dense matter at supranuclear densities determine the static and dynamical behavior of stellar matter [1-5]. The study of matter at extreme densities and temperatures has received renewed interest due to the possibility of attaining such conditions in relativistic heavy-ion collisions at GSI, and in the near future at CERN and Brookhaven.

It is believed that at extremely high densities, deconfinement will take place resulting in a transition from hadronic to quark matter. The transition point and its characteristics will depend crucially on the equation of state of matter in the hadronic phase. It is well known that the presence of strangeness, in the form of hyperons $(\Lambda, \Sigma)$ or mesons $\left(K^{-}\right)$, will soften the equation of state and will delay the transition. Most investigations up to date have been made in the framework of the mean field approach, either relativistic [6,7] or nonrelativistic, with effective Skyrme interactions [8]. Microscopic theories, on the other hand, aim at obtaining the properties of hadrons in dense matter from the bare free space interaction. In this sense, Brueckner theory was developed a long time ago and successfully helped to understand the properties of (nonstrange) nuclear matter starting from interactions that reproduce a huge amount of $N N$ scattering observables. A first attempt to incorporate strangeness in the form of hyperons within Brueckner theory was made in Refs. $[9,10]$, the latter extended to investigations of beta-stable nuclear matter [11]. A missing ingredient in these works was the hyperon-hyperon $(Y Y)$ interaction and the results of single-particle potentials or binding energy per baryon with a finite amount of hyperons were simply orientative.

The recent availability of a baryon-baryon potential [12] covering the complete $\mathrm{SU}(3) \times \mathrm{SU}(3)$ sector has allowed us to incorporate the $Y Y$ potential in a microscopic calculation of dense matter with nonzero hyperon fraction [13]. The incorporation of all possible baryon-baryon interactions required the solution of the $G$-matrix equation in coupled channels for different strangeness sectors: $N N(S=0), Y N(S=$
-1), $Y Y(S=-2,-3$, and -4$)$. The work of Ref. [13] concentrated mainly on isospin-saturated systems, i.e., systems with the same fraction of particles within the same isospin and strangeness multiplet: $T=1 / 2, S=0$ (neutrons and protons), $T=0, S=-1(\Lambda), T=1, S=-1\left(\Sigma^{-}, \Sigma^{0}, \Sigma^{+}\right)$, and $T=1 / 2, S=-2\left(\Xi^{-}, \Xi^{0}\right)$. In this way, the complications associated with different Fermi seas for each species of the same isospin-strangeness multiplet were avoided and the $G$ matrix in each sector was independent of the third component of isospin.

It is well known, however, that the presence of electrons causes nuclear star matter to be equilibrated against the weak $\beta$-decay reactions for neutron fractions much larger (a factor of 10 or more) than that for protons [14-16]. Also, the increase of negatively charged leptons with baryonic density will turn into a decrease when the appearance of negatively charged baryons becomes energetically more favorable. This is the case of the $\Sigma^{-}$hyperon, since neutralizing the proton charge with $\Sigma^{-}$instead of $e^{-}$will remove two energetic neutrons $\left(p \Sigma^{-} \leftrightarrow n n\right)$ instead of one $\left(p e^{-} \leftrightarrow n\right)$. It is clear, therefore, that a microscopic study of $\beta$-stable nuclear matter with hyperons requires the treatment of highly asymmetric matter, both in the nonstrange sector (protons vs neutrons) and the hyperonic one $\left(\Sigma^{-}\right.$vs $\Sigma^{0}$ and $\left.\Sigma^{+}\right)$. In the present paper we extend the study of Ref. [13] to allow for different fractions of each species. We will also explore the effect of the recently available $Y Y$ interaction on the single-particle potential of the hyperons, a crucial ingredient to determine the baryonic density at which the different hyperons appear. Our aim is to present a thorough analysis of the properties of the different baryons in dense matter, taking into account their mutual interactions. We will explore different baryonic densities and compositions that are relevant in the study of neutron stars.

\section{FORMALISM}

In this section we present the formalism to obtain, in the Brueckner-Hartree-Fock approximation, the single-particle 
energies of $n, p, \Lambda, \Sigma^{-}, \Sigma^{0}, \Sigma^{+}, \Xi^{-}$, and $\Xi^{0}$ embedded in an infinite system composed of different concentrations of such baryons. We first construct effective baryon-baryon $(B B)$ interactions ( $G$ matrices) starting from new realistic bare $B B$ interactions, which have become recently available for different strangeness channels [12].

\section{A. Effective $B B$ interaction}

The effective $B B$ interaction or $G$ matrix is obtained from the bare $B B$ interaction by solving the corresponding BetheGoldstone equation, which in partial wave decomposition and using the quantum numbers of the relative and centerof-mass motion (RCM) reads

$$
\begin{aligned}
& \left\langle\left(B_{3} B_{4}\right) k^{\prime \prime} K L^{\prime \prime} S^{\prime \prime}(J) T, M_{T}|G(\omega)|\left(B_{1} B_{2}\right) k K L S(J) T M_{T}\right\rangle \\
& =\left\langle\left(B_{3} B_{4}\right) k^{\prime \prime} K L^{\prime \prime} S^{\prime \prime}(J) T M_{T}|V|\left(B_{1} B_{2}\right) k K L S(J) T M_{T}\right\rangle+\sum_{L^{\prime}} \sum_{S^{\prime}} \sum_{B \widetilde{B}} \int k^{\prime 2} d k^{\prime}\left\langle\left(B_{3} B_{4}\right) k^{\prime \prime} K L^{\prime \prime} S^{\prime \prime}(J)\right. \\
& \left.\quad \times T M_{T}|V|(B \widetilde{B}) k^{\prime} K L^{\prime} S^{\prime}(J) T M_{T}\right\rangle \frac{\bar{Q}_{B \widetilde{B}}\left(k^{\prime}, K ; T, M_{T}\right)}{\omega-\frac{K^{2}}{2\left(M_{B}+M_{\widetilde{B}}\right)}-\frac{k^{\prime 2}\left(M_{B}+M_{\widetilde{B}}\right)}{2 M_{B} M_{\widetilde{B}}}-M_{B}-M_{\widetilde{B}}+i \eta}\left\langle(B \widetilde{B}) k^{\prime} K L^{\prime} S^{\prime}(J)\right. \\
& \left.\quad \times T M_{T}|G(\omega)|\left(B_{1} B_{2}\right) k K L S(J) T M_{T}\right\rangle .
\end{aligned}
$$

The starting energy $\omega$ corresponds to the sum of nonrelativistic single-particle energies of the interacting baryons including their rest masses. Note that we use the kinetic energy spectrum for the intermediate $B \widetilde{B}$ states. The variables $k, k^{\prime}$, $k^{\prime \prime}$ and $L, L^{\prime}, L^{\prime \prime}$ denote relative linear momenta and orbital momenta, respectively, while $K$ is the linear center-of-mass momentum. The total angular momentum, spin, isospin, and isospin projections are denoted by $J, S, T$, and $M_{T}$, respectively. As usual, $\bar{Q}_{B \tilde{B}}\left(k^{\prime}, K ; T, M_{T}\right)$ is the angle average of the Pauli operator which prevents the intermediate baryons $B$ and $\widetilde{B}$ from being scattered to states below their respective Fermi momenta $k_{F}^{(B)}$ and $k_{F}^{(\widetilde{B})}$. This angle average is shown in Appendix A, together with the expressions that define the Pauli operator in a particular $\left(T, M_{T}\right)$ channel in terms of the basis of physical states. Although we keep the index $M_{T}$ in the bare potential matrix elements, they do not really have a dependence on the third component of isospin since we consider charge symmetric and charge independent interactions. Therefore, the dependence of the $G$ matrix on the third component of isospin comes exclusively from the Pauli operator, since, as can be clearly seen in Appendix A, it acquires a dependence on $M_{T}$ when different concentrations of particles belonging to the same isomultiplet (i.e., different values for the corresponding $k_{F}$ 's) are considered.

In comparison with the pure nucleonic calculation, this problem is a little bit more complicated because of its coupled-channel structure. Whereas for the strangeness sectors 0 and -4 there is only one particle channel ( $N N$ and $\Xi \Xi$, respectively) and two possible isospin states $(T=0,1)$, in the $S=-1(S=-3)$ sector we are dealing with the $\Lambda N(\Lambda \Xi)$ and $\Sigma N(\Sigma \Xi)$ channels, coupled to $T=1 / 2$,

$$
\left(\begin{array}{ll}
G_{\Lambda N \rightarrow \Lambda N} & G_{\Lambda N \rightarrow \Sigma N} \\
G_{\Sigma N \rightarrow \Lambda N} & G_{\Sigma N \rightarrow \Sigma N}
\end{array}\right) \quad\left(\begin{array}{ll}
G_{\Lambda \Xi \rightarrow \Lambda \Xi} & G_{\Lambda \Xi \rightarrow \Sigma \Xi} \\
G_{\Sigma \Xi \rightarrow \Lambda \Xi} & G_{\Sigma \Xi \rightarrow \Sigma \Xi}
\end{array}\right),
$$

and the $\Sigma N(\Sigma \Xi)$ channel in isospin $T=3 / 2$ :

$$
\left(G_{\Sigma N \rightarrow \Sigma N}\right)\left(G_{\Sigma \Xi \rightarrow \Sigma \Xi}\right) .
$$

In the $S=-2$ sector we must consider the channels $\Lambda \Lambda$, $\Lambda \Sigma, \Xi N$, and $\Sigma \Sigma$ in isospin states $T=0$,

$$
\left(\begin{array}{ccc}
G_{\Lambda \Lambda \rightarrow \Lambda \Lambda} & G_{\Lambda \Lambda \rightarrow \Xi N} & G_{\Lambda \Lambda \rightarrow \Sigma \Sigma} \\
G_{\Xi N \rightarrow \Lambda \Lambda} & G_{\Xi N \rightarrow \Xi N} & G_{\Xi N \rightarrow \Sigma \Sigma} \\
G_{\Sigma \Sigma \rightarrow \Lambda \Lambda} & G_{\Sigma \Sigma \rightarrow \Xi N} & G_{\Sigma \Sigma \rightarrow \Sigma \Sigma}
\end{array}\right),
$$

$T=1$,

$$
\left(\begin{array}{lll}
G_{\Xi N \rightarrow \Xi N} & G_{\Xi N \rightarrow \Lambda \Sigma} & G_{\Xi N \rightarrow \Sigma \Sigma} \\
G_{\Lambda \Sigma \rightarrow \Xi N} & G_{\Lambda \Sigma \rightarrow \Lambda \Sigma} & G_{\Lambda \Sigma \rightarrow \Sigma \Sigma} \\
G_{\Sigma \Sigma \rightarrow \Xi N} & G_{\Sigma \Sigma \rightarrow \Lambda \Sigma} & G_{\Sigma \Sigma \rightarrow \Sigma \Sigma}
\end{array}\right)
$$

and $T=2$,

$$
\left(G_{\Sigma \Sigma \rightarrow \Sigma \Sigma}\right)
$$

In addition, each box $G_{B_{1} B_{2} \rightarrow B_{3} B_{4}}$ has a $2 \times 2$ matrix substructure to incorporate the couplings between $(L, S)$ states having the same total angular momentum $J$. This submatrix reads 


$$
\left(\begin{array}{ll}
\langle L=J, S=0|G| L=J, S=0\rangle & \langle L=J, S=0|G| L=J, S=1\rangle \\
\langle L=J, S=1|G| L=J, S=0\rangle & \langle L=J, S=1|G| L=J, S=1\rangle
\end{array}\right)
$$

for spin-singlet-spin-triplet coupling $(L=J, S=0 \leftrightarrow L=J, S=1)$ and

$$
\left(\begin{array}{cc}
(\langle L=J-1, S=1|G| L=J-1, S=1\rangle & \langle L=J-1, S=1|G| L=J+1, S=1\rangle \\
\langle L=J+1, S=1|G| L=J-1, S=1\rangle & \langle L=J+1, S=1|G| L=J+1, S=1\rangle
\end{array}\right)
$$

for tensor coupling $(L=J-1, S=1 \leftrightarrow L=J+1, S=1)$.

\section{B. Baryon single-particle energy in the Brueckner-Hartree-Fock approximation}

In the Brueckner-Hartree-Fock approximation the single-particle potential of a baryon $B_{1}$ which is embedded in the Fermi sea of baryons $B_{2}$ is given, using the partial wave decomposition of the $G$-matrix, by

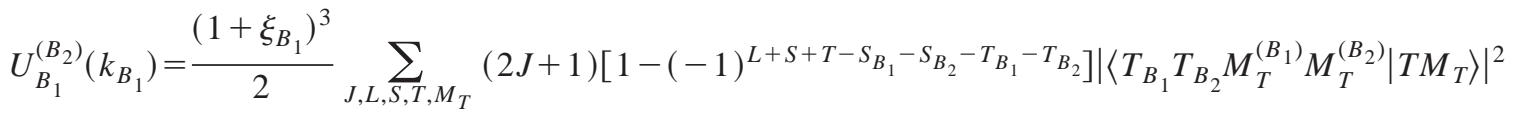

$$
\begin{aligned}
& \times \int_{0}^{k_{\max }} k^{2} d k f\left(k, k_{B_{1}}\right)\left\langle B_{1} B_{2} ; k K L S T M_{T}\left|G^{J}\left[E_{B_{1}}\left(k_{B_{1}}\right)+E_{B_{2}}\left(k_{B_{2}}\right)+m_{B_{1}}+m_{B_{2}}\right]\right| B_{1} B_{2} ; k K L S T M_{T}\right\rangle,
\end{aligned}
$$

if both types of baryons are identical, or by

$$
\begin{aligned}
U_{B_{1}}^{\left(B_{2}\right)}\left(k_{B_{1}}\right)= & \frac{\left(1+\xi_{B_{1}}\right)^{3}}{2} \sum_{J, L, S, T, M_{T}}(2 J+1)\left|\left\langle T_{B_{1}} T_{B_{2}} M_{T}^{\left(B_{1}\right)} M_{T}^{\left(B_{2}\right)} \mid T M_{T}\right\rangle\right|^{2} \int_{0}^{k_{\max }} k^{2} d k f\left(k, k_{B_{1}}\right) \\
& \times\left\langle B_{1} B_{2} ; k \text { KLSTM } M_{T}\left|G^{J}\left[E_{B_{1}}\left(k_{B_{1}}\right)+E_{B_{2}}\left(k_{B_{2}}\right)+m_{B_{1}}+m_{B_{2}}\right]\right| B_{1} B_{2} ; k K L S T M_{T}\right\rangle,
\end{aligned}
$$

if they are different. In the actual calculations, we consider all partial waves up to $J=4$. The labels $S_{B_{1}}, S_{B_{2}}\left(T_{B_{1}}, T_{B_{2}}\right)$ denote the spin (isospin) of baryons $B_{1}$ and $B_{2}$, respectively, and $\left\langle T_{B_{1}} T_{B_{2}} M_{T}^{\left(B_{1}\right)} M_{T}^{\left(B_{2}\right)} \mid T M_{T}\right\rangle$ is the Clebsch-Gordan coefficient coupling to total isospin $T$. The variable $k$ denotes the relative momentum of the $B_{1} B_{2}$ pair, which is constrained by

$$
k_{\max }=\frac{k_{F}^{\left(B_{2}\right)}+\xi_{B_{1}} k_{B_{1}}}{1+\xi_{B_{1}}},
$$

with $\xi_{B_{1}}=M_{B_{2}} / M_{B_{1}}$. Finally, the weight function $f\left(k, k_{B_{1}}\right)$, given by

$$
f\left(k, k_{B_{1}}\right)= \begin{cases}1 & \text { for } k \leqslant \frac{k_{F}^{\left(B_{2}\right)}-\xi_{B_{1}} k_{B_{1}}}{1+\xi_{B_{1}}}, \\ 0 & \text { for }\left|\xi_{B_{1}} k_{B_{1}}-\left(1+\xi_{B_{1}}\right) k\right|>k_{F}^{\left(B_{2}\right)}, \\ \frac{k_{F}^{\left(B_{2}\right) 2}-\left[\xi_{B_{1}} k_{B_{1}}-\left(1+\xi_{B_{1}}\right) k\right]^{2}}{4 \xi_{B_{1}}\left(1+\xi_{B_{1}}\right) k_{B_{1}} k} & \text { otherwise, }\end{cases}
$$

results from the analytical angular integration, once the angular dependence of the $G$-matrix elements is eliminated. This is done by choosing appropriate angular averages for the center of mass of the $B_{1} B_{2}$ pair and for the value of $k_{B_{2}}$ which enters in the determination of the starting energy. See Appendix B for details.

If the baryon $B_{i}$ is embedded in the Fermi seas of several baryons $B_{1}, B_{2}, B_{3}, \ldots$, including its own Fermi sea, then its single-particle potential is given by the sum of all the partial contributions,

$$
U_{B_{i}}(k)=\sum_{B_{j}} U_{B_{i}}^{\left(B_{j}\right)}(k)
$$

where $U_{B_{i}}^{\left(B_{j}\right)}(k)$ is the potential of the baryon $B_{i}$ due to the Fermi sea of baryons $B_{j}$. In this expression $k$ denotes the single-particle momentum of particle $B_{i}$. The nonrelativistic single-particle energy of baryon $B$ is then given by 


$$
E_{B}(k)=\frac{\hbar^{2} k^{2}}{2 M_{B}}+U_{B}(k) .
$$

This is precisely the single-particle energy that determines the value of the starting energy $\omega$ at which the $G_{B_{1} B_{2} \leftrightarrow B_{3} B_{4}}$ matrix in Eq. (2) [or (3)] should be evaluated. This implies a self-consistent solution of Eqs. (1), (2) [or (3)], and (7). The Fermi energy of each species is determined by setting $k$ to the corresponding Fermi momentum in the above expression.

\section{Energy density and binding energy per baryon}

The total nonrelativistic energy density $\varepsilon$ and the total binding energy per baryon, $B / A$, can be evaluated from the baryon single-particle potentials in the following way:

$$
\begin{gathered}
\varepsilon=2 \sum_{B} \int_{0}^{k_{F}^{(B)}} \frac{d^{3} k}{(2 \pi)^{3}}\left(\frac{\hbar^{2} k^{2}}{2 M_{B}}+\frac{1}{2} U_{B}(k)\right), \\
\frac{B}{A}=\frac{\varepsilon}{\rho},
\end{gathered}
$$

where $\rho$ is the total baryonic density. The density of a given baryon species is given by

$$
\rho_{B}=\frac{k_{F_{B}}^{3}}{3 \pi^{2}}=x_{B} \rho,
$$

where $x_{B}=\rho_{B} / \rho$ is the fraction of baryon $B$, which is of course constrained by

$$
\sum_{B} x_{B}=1
$$

\section{RESULTS}

We start this section by presenting results for the singleparticle potential of each baryon species, as a function of the baryon momentum, for several baryonic densities and various nucleonic and hyperonic fractions. We have focused on results for the Nijmegen model (e) of the recent parametrization [12], since it gives, together with model (f), the best predictions for hypernuclear observables [17], apart from reproducing the $Y N$ scattering scattering data as well as the other models. We will restrict our calculations to matter composed of neutrons, protons, $\Lambda^{\prime}$ 's and $\Sigma^{-}$'s, since these last two hyperons species are the first ones to appear as the density of $\beta$-stable neutron star matter increases [11]. This is confirmed on the recent study of [18] where, up to the density $1.2 \mathrm{fm}^{-3}$ considered there, $\Xi^{-}$and $\Xi^{0}$ baryons are absent.

In Fig. 1 we show our results for nonstrange nuclear matter at normal density, $\rho_{0}=0.17 \mathrm{fm}^{-3}$, and three proton fractions $\left(x_{p}=0.5 x_{N}, 0.25 x_{N}\right.$, and 0$)$, where $x_{N}$ is the fraction of nonstrange baryons, which in this case is 1 . We also show the hyperon single-particle potentials, denoted with the label "old," obtained with the Nijmegen 1989 version of the $Y N$ interaction [19]. In the right panel, corresponding to symmet-

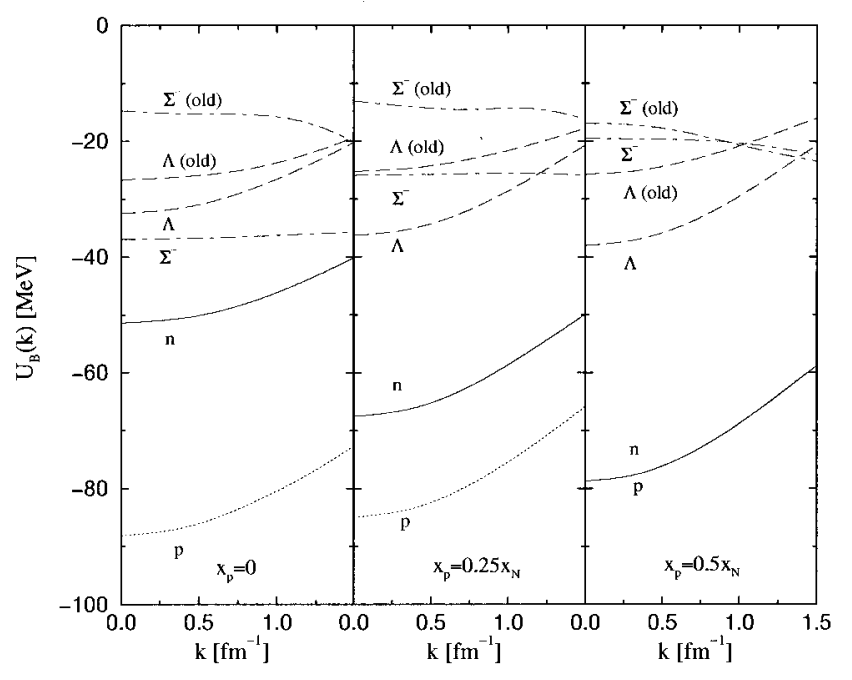

FIG. 1. Momentum dependence of the single-particle potentials for the different species at $\rho=0.17 \mathrm{fm}^{-3}$, hyperon fraction $x_{Y}=0$, and several nucleon asymmetries.

ric nuclear matter, we see that neutrons and protons have the same single-particle potential, of the order of $-79 \mathrm{MeV}$ at zero momentum. Looking at the middle and left panels we see how, as the fraction of protons decreases, the protons gain binding while the neutrons lose attraction. This is a consequence of the different behavior of the $N N$ interaction in the $T=0$ and $T=1$ channels, the $T=0$ channel being substantially more attractive. The potential of the proton is built from more $T=0$ than $T=1$ pairs and hence becomes more attractive. The $\Lambda$ single-particle potential in symmetric nuclear matter turns out to be around $-38 \mathrm{MeV}$ at $k=0$ and has a smooth parabolic behavior as a function of $k$. This result is larger than the value of $-30 \mathrm{MeV}$ obtained when one extrapolates to large $A$ the $s$-wave $\Lambda$ single-particle energy of several hypernuclei [20]. It is also much larger in magnitude than the value of around $-24 \mathrm{MeV}$ [21-23] which is obtained using the 1989 version of the Nijmegen $Y N$ potential [19] with the standard choice for the spectrum of the intermediate $Y N$ states in the Bethe-Goldstone equation. The value of the $\Sigma$ single-particle potential at $k=0$ of $-20 \mathrm{MeV}$ is somewhat more attractive than that obtained with the 1989 potential of around $-17 \mathrm{MeV}$. The function $U_{\Sigma^{-}}(k)$ remains pretty constant in the range of momenta explored. Apart from the different size, the new singleparticle hyperon potentials also show a totally different behavior with increasing asymmetry than that observed for the potentials obtained with the 1989 Nijmegen $Y N$ interaction. While the old $\Lambda$ single-particle potential turns to be slightly more attractive with increasing neutron fraction (i.e., going from the right panel to the left one), the new one becomes slightly more repulsive. The changes for the $\Sigma^{-}$singleparticle potential are more drastic. While the 1989 interaction gives a $\Sigma^{-}$potential which shows a little change with increasing neutron fraction, the new $\Sigma^{-}$potential becomes strongly attractive. The value at $k=0$ for the $\Sigma^{-}$potential changes from about $-20 \mathrm{MeV}$ in symmetric nuclear matter to $-37 \mathrm{MeV}$ in neutron matter. This has important consequences in the composition of dense matter: if hyperons feel 


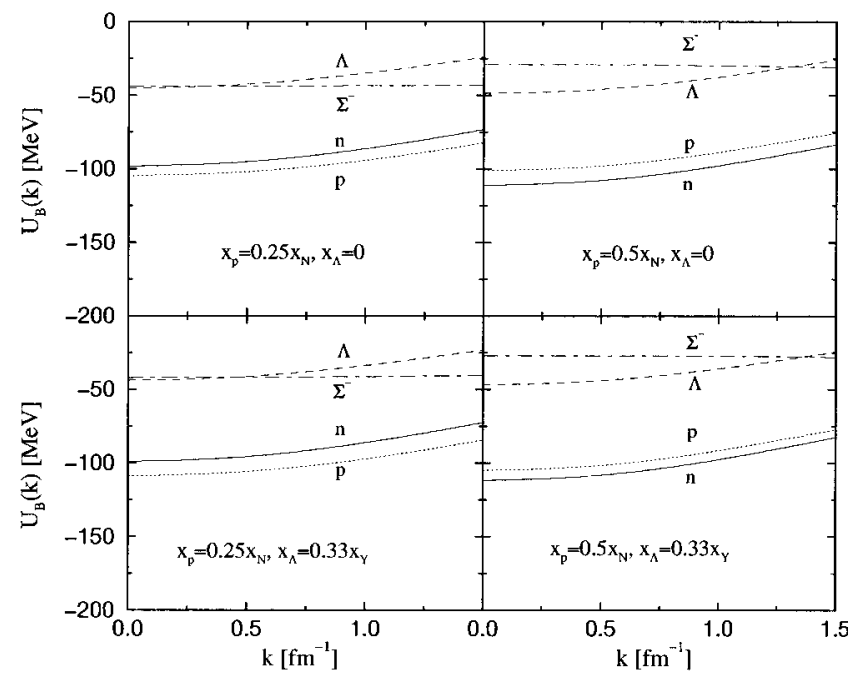

FIG. 2. Momentum dependence of the single-particle potentials for the different species at $\rho=0.3 \mathrm{fm}^{-3}$ and hyperon fraction $x_{Y}$ $=0.1$. The right panels correspond to symmetric nuclear matter, $x_{n}=x_{p}=0.5 x_{N}$, while the left ones are for asymmetric nuclear matter with $x_{n}=3 x_{p}=0.75 x_{N}$. In the top panels the hyperonic fraction is built exclusively from $\Sigma^{-}\left(x_{\Sigma^{-}}=x_{Y}\right)$ while in the bottom ones there is a fraction of $\Lambda^{\prime}$ 's $\left(x_{\Lambda}=x_{Y} / 3\right)$ and $\Sigma^{-}$'s $\left(x_{\Sigma^{-}}=2 x_{Y} / 3\right)$.

substantially more attraction, their appearance in dense matter will happen at lower density. We note that our results with the 1989 Nijmegen interaction are consistent with those shown in [11], where the same $Y N$ interaction is used. Some differences are found in the magnitude of the single-particle potentials which should be ascribed to the use of a continuum spectrum prescription in the case of [11].

Having established how the nucleons affect the singleparticle potential of hyperons it is necessary to investigate the influence of a finite fraction of hyperons on the hyperons themselves and on the nucleons. This is visualized in Figs. 2 and 3 that show the single-particle potentials of the different baryons as functions of the momentum. Figure 2 shows results at $\rho=0.3 \mathrm{fm}^{-3}$ and a hyperon fraction $x_{Y}=0.1$, which

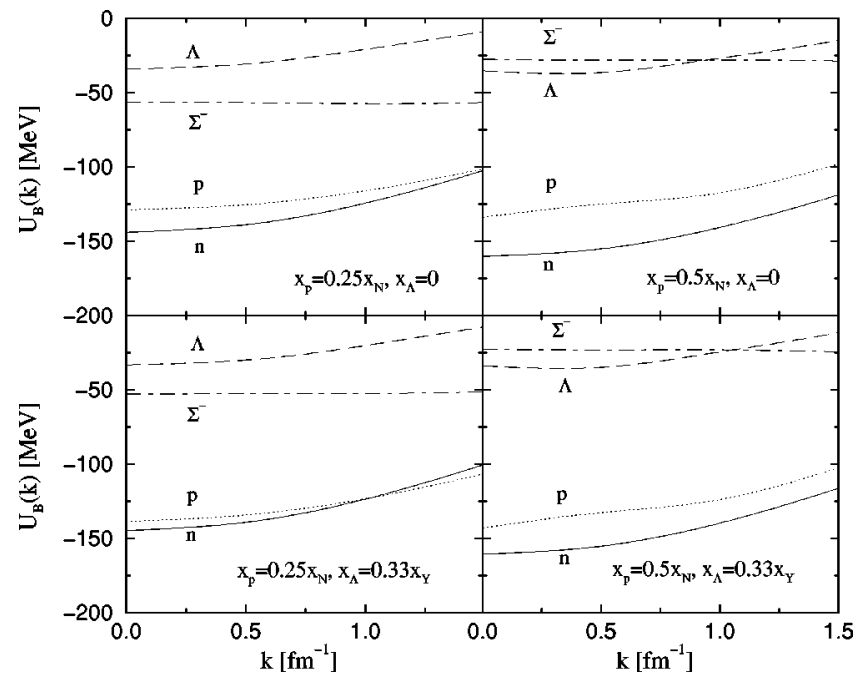

FIG. 3. The same as Fig. 2 for a baryon density $\rho=0.6 \mathrm{fm}^{-3}$. is assumed to come from only $\Sigma^{-}$(top panels) or split into $\Sigma^{-}$and $\Lambda$ hyperons in a proportion $2: 1$, hence $x_{\Sigma^{-}}=2 x_{Y} / 3$ and $x_{\Lambda}=x_{Y} / 3$ (bottom panels). The panels on the right correspond to symmetric proton-neutron composition $\left(x_{p}=x_{n}\right.$ $=0.5 x_{N}$, where $\left.x_{N}=0.9\right)$ and the ones on the left correspond to a higher proportion of neutrons $\left(x_{p}=0.25 x_{N}, x_{n}\right.$ $\left.=0.75 x_{N}\right)$. Starting at the upper-right panel we observe that the presence of $\Sigma^{-}$hyperons already breaks the symmetry between the proton and neutron single-particle potentials in a symmetric nucleonic composition, the neutrons feeling around $-10 \mathrm{MeV}$ more attraction. This is due to a different behavior between the $\Sigma^{-} n$ interaction which only happens via the attractive $T=3 / 2$ channel and the $\Sigma^{-} p$ interaction that also receives contributions from the very repulsive $T$ $=1 / 2 \Sigma N$ component. In fact, the difference between the neutron and proton potentials is not as pronounced as we move to the lower panel on the right, where some $\Sigma^{-}$hyperons are replaced by $\Lambda$ hyperons which act identically over protons and neutrons. In the upper left panel, where we have increased the neutron fraction in the nonstrange sector, we observe the typical pattern for the nucleon single-particle potentials commented on Fig. 1: the particle with the smallest fraction (i.e., the proton) shows more binding. However, this behavior is partially compensated by the presence of a sea of $\Sigma^{-}$which provides attraction (repulsion) to the neutron (proton) single-particle potential. We also observe that the $\Sigma^{-}$ feels more attraction, as a consequence of having replaced some repulsive $\Sigma^{-} p$ pairs by attractive $\Sigma^{-} n$ ones. The $\Lambda$ loses binding because the Fermi sea of neutrons is larger and their contribution to the $\Lambda$ single-particle energy explores higher relative momentum components of the effective $\Lambda n$ interaction, which are less attractive than the small relative momentum ones. Finally, since the Fermi sea of hyperons is small, the differences observed in the potentials by going from the top panels to the corresponding lower ones (which amounts to replacing $\Sigma^{-}$hyperons by $\Lambda$ ones) are also small.

Similar effects are found in the results reported in Fig. 3, which have been obtained for a baryonic density $\rho$ $=0.6 \mathrm{fm}^{-3}$, where it is expected that nuclear matter in $\beta$ equilibrium already contains hyperons [11]. The singleparticle potential of the $\Lambda$ hyperon is less attractive than that for $\rho=0.3 \mathrm{fm}^{-3}$ while that of the $\Sigma^{-}$is very similar. It just gains somewhat more attraction when the number of neutrons increase relative to that of protons in going from the right panels to the left ones. As for the nucleon singleparticle potentials we observe, also in the left panels, that the attractive $\Sigma^{-} n$ interaction is enhanced at these high densities and makes the neutron spectrum more attractive than the proton one, even in the asymmetric situation when one would expect the protons to be more bound.

To assess the influence of the $Y Y$ interaction we represent the separate contributions building the $\Lambda$ single-particle potential in Fig. 4 and those for the $\Sigma^{-}$one in Fig. 5, for a baryonic density of $0.6 \mathrm{fm}^{-3}$. The hyperon fraction of $x_{Y}$ $=0.1$ is split into fractions $x_{\Sigma^{-}}=2 x_{Y} / 3$ and $x_{\Lambda}=x_{Y} / 3$ for $\Sigma^{-}$ and $\Lambda$ hyperons, respectively. The results on the right-hand side of Figs. 4 and 5 correspond to the symmetric nuclear 


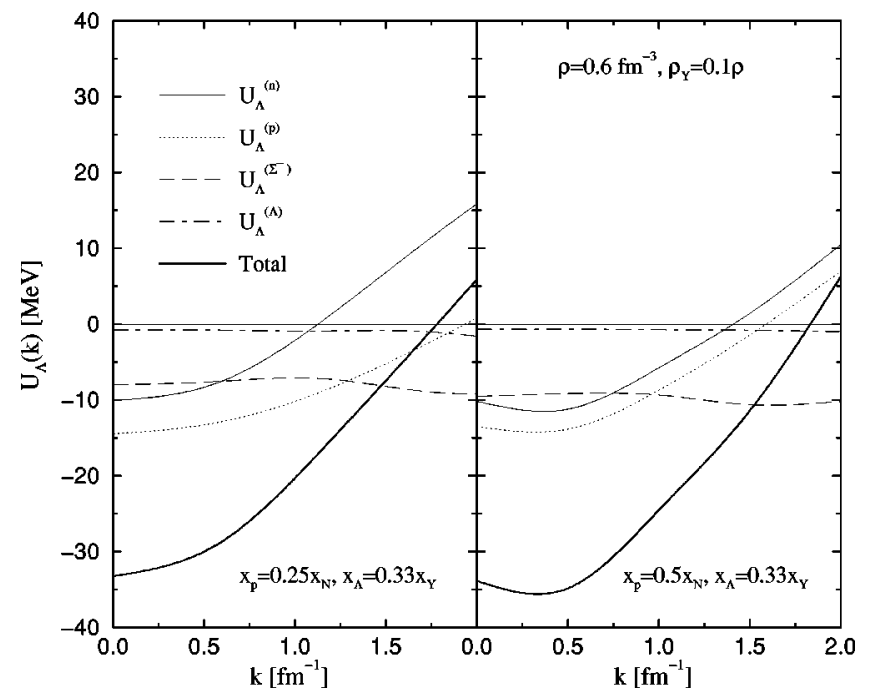

FIG. 4. Separate contributions of each species to the $\Lambda$ singleparticle potential at $\rho=0.6 \mathrm{fm}^{-3}$ and hyperon fraction $x_{Y}=0.1 \mathrm{split}$ into $x_{\Sigma^{-}}=2 x_{Y} / 3$ and $x_{\Lambda}=x_{Y} / 3$. The right panel is for symmetric nuclear matter $\left(x_{n}=x_{p}=0.5 x_{N}\right)$ and the left one for asymmetric nuclear matter $\left(x_{n}=3 x_{p}=0.75 x_{N}\right)$.

case and those on the left to a neutron fraction 3 times larger than that of protons. We see that the contribution to the $\Lambda$ potential from the $\Lambda$ hyperons, represented by the dashdotted line, is attractive and almost negligible, due to a weak attractive $\Lambda \Lambda$ effective interaction [13] and to the small amount of $\Lambda$ particles present. On the contrary, the contribution from the $\Sigma^{-}$hyperons is larger, of the order of -10 $\mathrm{MeV}$ in nuclear-symmetric matter and slightly less in nuclear-asymmetric one, which is comparable in size with the contribution from protons and neutrons. This example clearly shows the important role of the $Y Y$ interaction in modifying the properties of the $\Lambda$ hyperon. The $\Lambda$ acquires more attraction and its appearance in dense matter becomes more favorable with respect to the situation in which the $Y Y$ interaction was neglected. The fact that the neutron (thin solid line) and proton (dotted line) contributions to the $\Lambda$

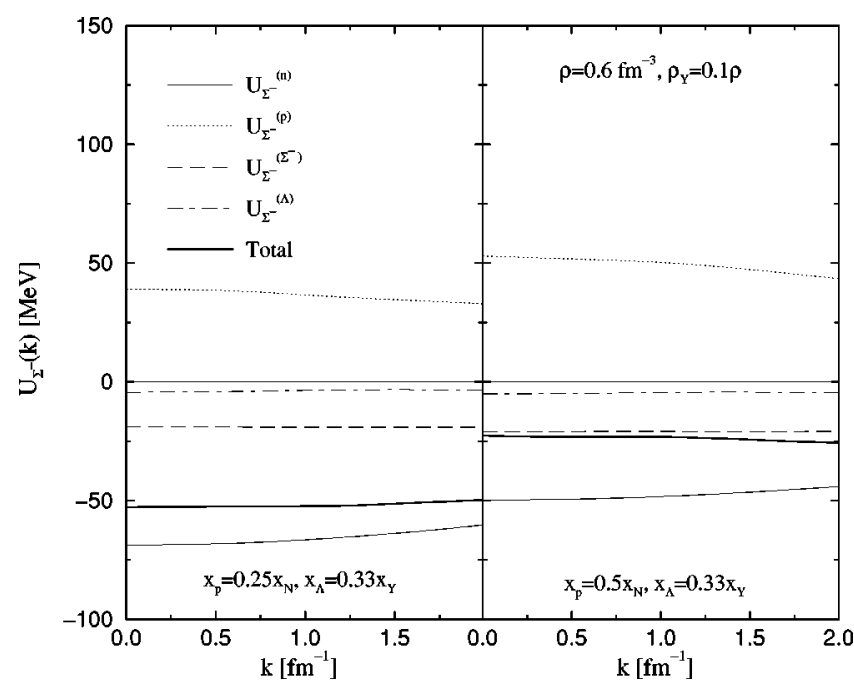

FIG. 5. The same as Fig. 4 for the $\Sigma^{-}$single-particle potential.

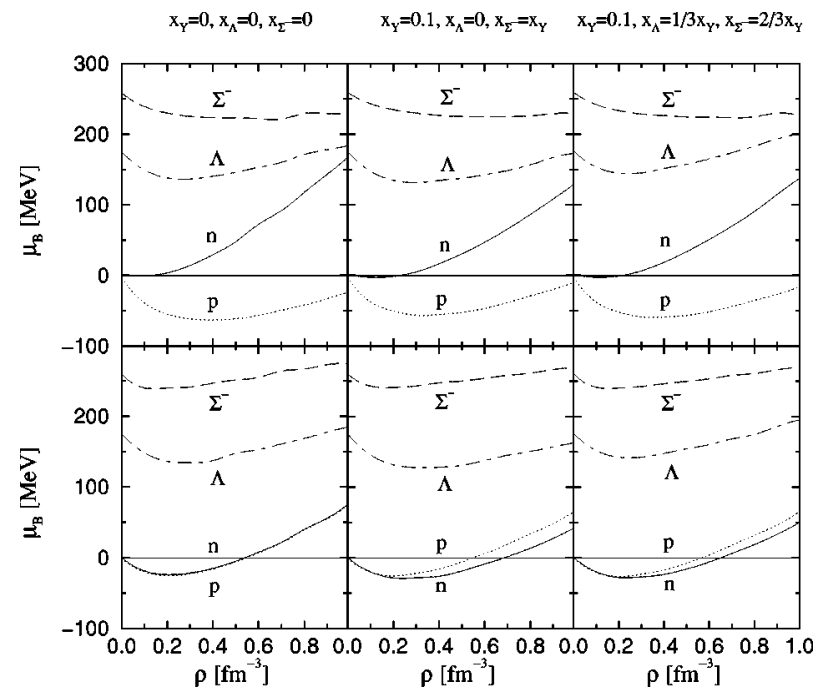

FIG. 6. Chemical potentials of the different species as functions of total baryonic density, for different nucleonic asymmetries and strangeness fractions. The top panels correspond to the asymmetric nuclear matter case $\left(x_{n}=3 x_{p}=0.75 x_{N}\right)$, while the bottom ones correspond to symmetric nuclear matter $\left(x_{n}=x_{p}=0.5 x_{N}\right)$.

single-particle potential are not the same in nuclearsymmetric matter is due to the $\Sigma^{-}$hyperons which make the neutrons feel more attraction and, consequently, the $\Lambda n$ pairs explore the effective $\Lambda N$ interaction at smaller energies, where it is less attractive. The different contributions to the $\Sigma^{-}$potential are shown in Fig. 5. The $\Lambda$ hyperons (dotdashed line) contribute very little due to the reduced value of their Fermi momentum. The contribution of the $\Sigma^{-} \Sigma^{-}$pairs (long-dashed line) is very important, of the order of -25 $\mathrm{MeV}$ in symmetric nuclear matter, and becomes crucial due to the fact that the neutron (thin solid line) and the proton (dotted line) contributions, which amount each one to about $50 \mathrm{MeV}$ in magnitude, almost cancel each other. In the left panel, the replacement of protons by neutrons, lowers the $\Sigma^{-}$ single-particle potential considerably, by about $25 \mathrm{MeV}$. Again, neglecting the $Y Y$ interactions here would have made the $\Sigma^{-}$potential about $20-25 \mathrm{MeV}$ less attractive.

The analysis of the structure of $\beta$-stable matter requires knowledge of the chemical potential $\left(\mu_{B}\right)$ of each baryon, defined at zero temperature as the single-particle energy of the Fermi momentum [Eq. (7)]. In Fig. 6 we show the chemical potentials as functions of density for different nucleon asymmetries and hyperon fractions. Note that the curves are measured with respect to the nucleon mass and contain, in addition to the nonrelativistic Fermi energy, the baryon mass of each species. The top panels show the results for asymmetric nuclear matter $\left(x_{n}=3 x_{p}=0.75 x_{N}\right)$ whereas the bottom panels stand for the symmetric case. In the left panels we show results for purely nucleonic matter $\left(x_{Y}=0\right)$, and in the central panels we have $x_{\Sigma^{-}}=x_{Y}=0.1$, while on the right panels $x_{Y}$ is distributed into $x_{\Lambda}=x_{Y} / 3$ and $x_{\Sigma^{-}}=2 x_{Y} / 3$. The behavior of the chemical potentials when increasing the nucleonic asymmetry as well as the hyperonic fraction follows closely the trends observed in Figs. 1, 2, and 3 for the single-particle potential at densities $\rho=0.17,0.3$, and 
$0.6 \mathrm{fm}^{-3}$, respectively. We just have to consider here that the curves in Fig. 6 also contain the kinetic energy of the corresponding Fermi momentum. It is interesting to comment on the high density behavior of the chemical potentials, since this will determine the feasibility of having hyperons in beta-stable neutron star matter. In symmetric nuclear matter, both the $\Lambda$ and the $\Sigma^{-}$chemical potentials show, from a certain density on, an increase with increasing density which is very mild as compared to that assumed by phenomenological $Y N$ interactions [24]. When the number of neutrons over that of protons is increased (top panels), the $\Lambda$ chemical potential barely changes because of the similarity between the $\Lambda n$ and $\Lambda p$ interaction. However, the $\Sigma^{-}$hyperon acquires more binding due to the dominant $\Sigma^{-} n$ attractive pairs over the $\Sigma^{-} p$ repulsive ones. This will favor the appearance of $\Sigma^{-}$in dense neutron star matter, through the $n n \rightarrow p \Sigma^{-}$conversion, when the equilibrium between chemical chemical potentials is achieved at both sides. Once a Fermi sea of $\Sigma^{-}$hyperons starts to build up, however, the neutrons become more attractive, moderating, in turn, the appearance of $\Sigma^{-}$hyperons. As we see, the composition of dense neutron star matter in equilibrium will result from a delicate interplay between the mutual influence among the different species. In fact, one needs to find, at each baryonic density, the particle fractions which balance the chemical potentials in the weak and strong reactions that transform the species among themselves. This study, which is beyond the scope of the present work, will be presented in a separate publication [18].

One of the novelties of this work is that we allow for different concentrations of the baryon species. Therefore, we can explicitly treat the dependence of the $G$ matrix on the third component of isospin which comes from the Pauli operator of species $B, \widetilde{B}$ that may have, even when belonging to the same isospin-strangeness multiplet, different Fermi momenta. See Appendix A for more details.

In Fig. 7 we report the diagonal $\Sigma N \rightarrow \Sigma N G$-matrix elements in the ${ }^{1} S_{0}$ channel, as a function of relative momentum for a density $\rho=0.6 \mathrm{fm}^{-3}$, taking $x_{\Lambda}=0$ and $x_{\Sigma^{-}}=x_{Y}$ $=0.1$. The top panels correspond to the isospin $T=1 / 2$ channel and the lower ones to the $T=3 / 2$ one. The panels on the right are for symmetric nuclear matter, $x_{n}=x_{p}=0.5 x_{N}$, while those on the left correspond to $x_{p}=0.25 x_{N}$. The starting energy and center of mass is the same for all the curves shown in the same plot; thus the dependence on $M_{T}$ comes exclusively from the Pauli operator. Note that different pairs of particles contribute to each $\left(T, M_{T}\right)$ combination. The case $\left(T, M_{T}\right)=(1 / 2,+1 / 2)$ receives contributions from $\Sigma^{+} n$ and $\Sigma^{0} p$ pairs while $\Sigma^{-} p$ and $\Sigma^{0} n$ contribute to $\left(T, M_{T}\right)$ $=(1 / 2,-1 / 2)$. In the case of isospin $T=3 / 2$ one has contributions from $\Sigma^{-} n\left(M_{T}=-3 / 2\right), \Sigma^{0} n, \Sigma^{-} p\left(M_{T}=-1 / 2\right)$, $\Sigma^{+} n, \Sigma^{0} p\left(M_{T}=+1 / 2\right)$, and $\Sigma^{+} p\left(M_{T}=+3 / 2\right)$. We observe that the curve corresponding to the third component $M_{T}$ less affected by Paui blocking is always more attractive as the phase space for intermediate states, which induce attractive corrections to the potential matrix elements, is larger. This is clearly seen in the top panel on the right, since the dotted line contains a channel with the $\Sigma^{-}$hyperon.

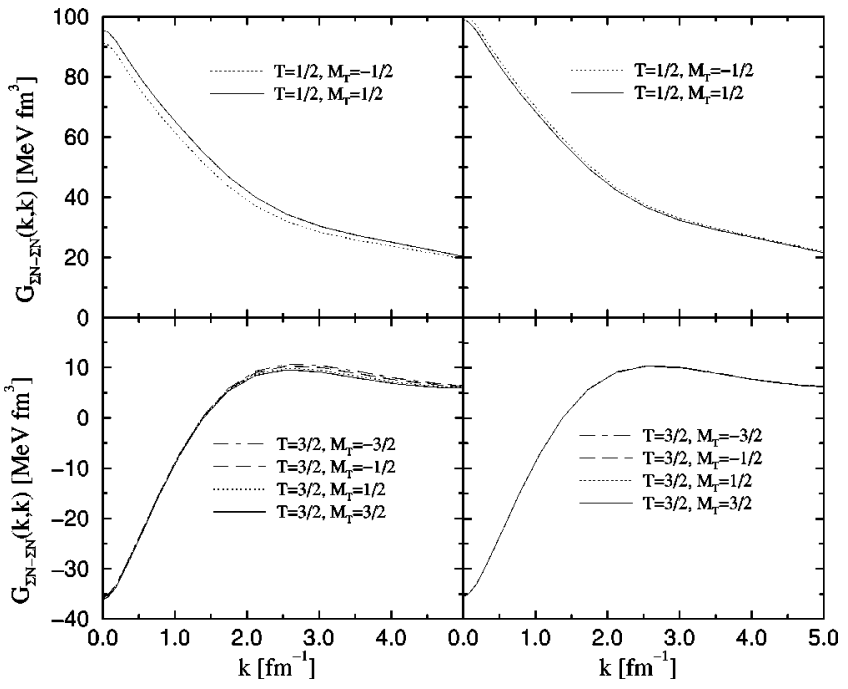

FIG. 7. Diagonal $\Sigma N G$ matrix in the ${ }^{1} S_{0}$ partial wave as a function of the relative momentum at a density $\rho=0.6 \mathrm{fm}^{-3}$, for the different $\left(T, M_{T}\right)$ isospin channels. The right panels are for symmetric nuclear matter, $x_{n}=x_{p}=0.5 x_{N}$, while the left ones correspond to $x_{n}=3 x_{p}=0.75 x_{N}$. In all cases $x_{\Sigma^{-}}=0.1$ and $x_{\Lambda}=0$.

When the nucleonic asymmetry is increased by going to the panel on the left, the effects of Pauli blocking on the neutrons are more important than those on the $\Sigma^{-}$hyperons. This is the reason for the solid curve to appear above the dotted one, since the $\left(T, M_{T}\right)=(1 / 2,+1 / 2)$ case receives contributions from $\Sigma^{0} p$ and $\Sigma^{+} n$ pairs in a proportion $1: 2$ and it contains relatively more neutrons than the case $\left(T, M_{T}\right)=(1 / 2,-1 / 2)$ with $\Sigma^{-} p$ and $\Sigma^{0} n$ pairs in a proportion 2:1. In the case of $T=3 / 2$ we observe that the asymmetry on the $\Sigma$ multiplet barely induces any dependence on $M_{T}$ in the $G$ matrix, as can be seen from the bottom panel on right. However, one can observe differences when going to asymmetric nuclear matter on the left panel since the Pauli blocking on $\Sigma^{-} n$ pairs $\left(M_{T}=-3 / 2\right)$ is enhanced over that on $\Sigma^{+} p$ pairs $\left(M_{T}=+3 / 2\right)$. As we can see, in all cases considered here the dependence of the $G$ matrix on the third component of the isospin is very weak and can almost be neglected. We have also encountered this weak dependence in the other $B \widetilde{B} G$ matrices. Therefore, a presumably good strategy and less time consuming would be to obtain the $G$ matrices in isospin-saturated systems and, afterwards, calculate the single-particle potentials by folding the "approximate" effective interactions with the different baryon Fermi seas.

We finish this section by reporting in Fig. 8 the binding energy per baryon as a function of density. The right and left figures describe symmetric and asymmetric $\left(x_{n}=3 x_{p}\right.$ $=0.75 x_{N}$ ) nuclear matter, respectively. In the top panels, we show the binding energy with $x_{\Sigma^{-}}=0$ for several values of $x_{\Lambda}$ while in the bottom panels we consider $x_{\Lambda}=0$ and vary the concentration of $\Sigma^{-}$hyperons. The binding energy per baryon, calculated according to Eqs. (8) and (9), is the result of a balance between the average kinetic energy of each baryon Fermi sea and the contribution from the mutual interactions, given by the average of the single-particle potential 


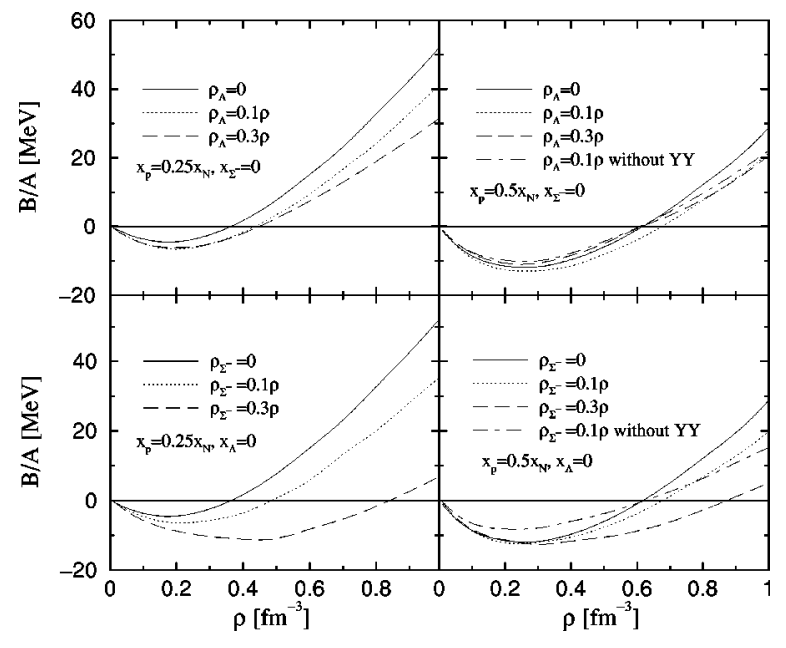

FIG. 8. Binding energy per baryon as a function of the baryon density. In the top panels we set $x_{\Sigma^{-}}=0$ and show results for several values of $x_{\Lambda}$, while the bottom panels correspond to $x_{\Lambda}=0$ and different fractions of $\Sigma^{-}$, s. The panels on the right are for symmetric nuclear matter, while the left ones correspond to asymmetric nuclear matter $\left(x_{n}=3 x_{p}=0.75 x_{N}\right)$. In the case of nuclear symmetric matter with $10 \%$ of hyperons we also show a curve (dash-dotted line) where the $Y Y$ interaction has been turned off.

of each species. In order to identify the effects of the $Y Y$ interaction on the binding energy per baryon we have also included a curve corresponding to a calculation with a $10 \%$ of hyperons (either $\Lambda^{\text {'s }}$ or $\Sigma^{-}$'s) where the $Y Y$ interaction is turned off (dash-dotted line). In both cases, turning the $Y Y$ interaction on results in a gain of binding energy which is larger in the case of $\Sigma^{-}$. The binding energy per baryon shows a saturation density, i.e., a density for which the thermodynamic pressure is zero, which is too high when we consider the composition with only nucleons. The location of this saturation density is little affected when the percentage of hyperons is increased. When a small amount of nucleons is substituted by hyperons there is automatically a decrease of the kinetic energy contribution because the hyperons can be accomodated in lower momentum states and in addition have a larger bare mass. The analysis of the influence of the effective interaction on the binding energy must be made separately for $\Lambda$ 's and $\Sigma^{-}$'s. Although the effective $\Lambda N$ and $\Lambda \Lambda$ interactions are clearly less attractive than the $N N$ one, the reduction of kinetic energy is clearly enough to compensate for the loss of binding energy when a $10 \%$ of nucleons is substituted by $\Lambda$ 's. Notice, however, that we have to consider the $\Lambda \Lambda$ interaction in order to obtain this increase of binding with respect to the pure nucleonic case. At $x_{\Lambda}$ $=30 \%$ the loss of kinetic energy is not enough to compensate for the loss of attraction from the effective interactions and less binding energy than the case with only nucleonic degrees of freedom is obtained. Looking at the lower panels for the $\Sigma^{-}$hyperons we observe that the binding energy per baryon gains more attraction as compared to the case for $\Lambda$ 's. This is due, essentially, to the larger loss of kinetic energy due to the larger mass of the $\Sigma^{-}$. In general, the replacement of nucleons by hyperons produces a gain in binding energy and a softening of the equation of state. The appearance of hyperons in beta-stable matter, the softening of the equation of state, and its implications on the properties of neutron stars are deferred to a future study [18].

\section{CONCLUSIONS}

In this work we have developed the formalism for microscopic Brueckner-type calculations of dense nuclear matter with strangeness, allowing for any concentration of the different baryon species.

By relating the Pauli operator to the different pairs of physical particles that contribute to the particular $\left(T, M_{T}\right)$ channel (see appendix A), we have been able to obtain the $M_{T}$ dependence of the effective interaction ( $G$ matrix) between any two species.

We have seen, however, that the dependence of the $G$ matrix on the third component of isospin is weak enough to allow, in future studies, for a simpler strategy consisting of obtaining the effective interactions in isospin-saturated situations $\left(k_{F}^{(n)}=k_{F}^{(p)}, k_{F}^{\left(\Sigma^{-}\right)}=k_{F}^{\left(\Sigma^{0}\right)}=k_{F}^{\left(\Sigma^{+}\right)}, k_{F}^{\left(\Xi^{-}\right)}=k_{F}^{\left(\Xi^{0}\right)}\right)$. The various single-particle potentials can then be obtained by folding the approximate effective interactions with the Fermi seas of the different species.

We have studied the dependence of the single-particle potentials on the nucleon and hyperon asymmetries, focusing on situations that can be relevant in future studies of betastable neutron star matter with strangeness. This is why, apart from neutrons and protons, we have only considered the $\Lambda$ and $\Sigma^{-}$hyperons, which are the first ones expected to appear. We have compared the symmetric nuclear matter composition $\left(x_{n}=x_{p}=x_{N}\right)$ with the asymmetric case containing a large fraction of neutrons $\left(x_{n}=3 x_{p}=0.75 x_{N}\right)$, for a small, but relevant, hyperon fraction $x_{Y}=0.1$. This fraction may be fully composed by $\Sigma^{-}$hyperons $\left(x_{\Sigma^{-}}=x_{Y}\right)$ or contain also a small proportion of $\Lambda$ 's $\left(x_{\Sigma^{-}}=2 x_{\Lambda}=2 x_{Y} / 3\right)$. We find that the presence of hyperons, especially $\Sigma^{-}$, modifies substantially the single-particle potentials of the nucleons. The neutrons feel an increased attraction due to the $\Sigma^{-} n$ effective interaction that only happens through the very attractive $T=3 / 2 \sum N$ channel, while the protons feel a repulsion as the $\Sigma^{-} p$ pairs also receive contributions from the very repulsive $T=1 / 2 \Sigma N$ one.

By decomposing the $\Lambda$ and $\Sigma^{-}$single-particle potentials in the contributions from the various species, we have seen the relevance of considering the $Y Y$ interaction. For a baryonic density of $0.6 \mathrm{fm}^{-3}$, a nuclear asymmetry of $x_{n}=3 x_{p}$ $=0.75 x_{N}$, and a hyperon fraction of $x_{Y}=0.1$ (split into $x_{\Sigma^{-}}=2 x_{Y} / 3$ and $\left.x_{\Lambda}=x_{Y} / 3\right)$, we find that the hyperonic contribution to the $\Lambda$ single-particle potential at zero momentum is of the order of $-10 \mathrm{MeV}\left[1 / 3\right.$ of the total $\left.U_{\Lambda}(0)\right]$ and that for the $\Sigma^{-}$is of the order of $-25 \mathrm{MeV}[1 / 2$ of the total $\left.U_{\Sigma-}(0)\right]$.

In the absence of hyperonic Fermi seas the $\Lambda$ and $\Sigma^{-}$ chemical potentials show a mild increase with increasing baryonic density. The presence of a Fermi sea of $\Sigma^{-}$hyperons slows down this increase, especially for the $\Sigma^{-}$chemical potential and in the case of asymmetric nuclear matter, due to the very attractive $T=3 / 2 \Sigma N$ interaction acting on $\Sigma^{-} n$ 
pairs. This will make the balance between chemical potentials in the strong $n n \rightarrow \Sigma^{-} p$ conversion easier and will favor the appearance of $\Sigma^{-}$at lower densities.

Finally, we have studied the modifications of the binding energy per baryon in symmetric and asymmetric nuclear matter when some nucleons are replaced either by $\Lambda$ or $\Sigma^{-}$ hyperons. As expected, we observe an increase in the binding energy, which increases with density, mainly as a result of a decrease in kinetic energy because the hyperons can be accommodated in lower momentum states and have a larger mass. This effect will produce a softening in the equation of state that will influence the behavior of dense matter and the structure of neutron stars.

\section{ACKNOWLEDGMENTS}

We would like to thank H.J. Schulze for useful comments. This work is partially supported by the DGICYT Contract No. PB95-1249 (Spain) and by the Generalitat de Catalunya Grant No. 1998SGR-11. One of the authors (I.V.) wishes to acknowledge support from the Ministerio de Educación y Cultura (Spain).

\section{APPENDIX A: PAULI OPERATOR IN THE DIFFERENT STRANGENESS CHANNELS}

In this appendix we show how the Pauli operator $Q_{B \tilde{B}}$, which prevents scattering into occupied $B \widetilde{B}$ intermediate states, acquires a dependence on the third component of isospin due to the different Fermi momenta of baryons $B$ and $\widetilde{B}$. The Pauli operator reads

$$
Q_{B \widetilde{B}}(\vec{k}, \vec{K})= \begin{cases}1 & \text { for }|\alpha \vec{K}+\vec{k}|>k_{F}^{B} \text { and }|\beta \vec{K}-\vec{k}|>k_{F}^{\tilde{B}}, \\ 0 & \text { otherwise, }\end{cases}
$$

where $\vec{k}$ and $\vec{K}$ are, respectively, the relative and total momenta of the $B \widetilde{B}$ pair, $\alpha=m_{B} /\left(m_{B}+m_{\tilde{B}}\right)$ and $\beta=m_{\widetilde{B}} /\left(m_{B}\right.$ $\left.+m_{\tilde{B}}\right)$. In order to solve the Bethe-Goldstone equation in partial wave representation [see Eq. (1)] we need to perform an angle average of the Pauli operator, which reads

$$
\bar{Q}_{B \widetilde{B}}(k, K)=\left\{\begin{array}{cl}
\frac{1}{2}\left(\cos \theta_{B}+\cos \theta_{\widetilde{B}}\right) & \text { if } \cos \theta_{B}+\cos \theta_{\widetilde{B}}>0, \\
0 & \text { if } \cos \theta_{B}+\cos \theta_{\widetilde{B}}<0,
\end{array}\right.
$$

where

$$
\cos \theta_{B}= \begin{cases}1 & \text { if }|\alpha \vec{K}-\vec{k}|>k_{F}^{(B)}, \\ \frac{\alpha^{2} K^{2}+k^{2}-k_{F}^{(B) 2}}{2 \alpha K k} & \text { otherwise, }\end{cases}
$$

and

$$
\cos \theta_{\tilde{B}}= \begin{cases}1 & \text { if }|\beta \vec{K}-\vec{k}|>k_{F}^{(\widetilde{B})}, \\ \frac{\beta^{2} K^{2}+k^{2}-k_{F}^{(\widetilde{B}) 2}}{2 \beta K k} & \text { otherwise. }\end{cases}
$$

Taking the following convention for the isospin states representing the particle basis:

$$
\begin{gathered}
|n\rangle=|1 / 2,-1 / 2\rangle ;|p\rangle=|1 / 2,+1 / 2\rangle, \\
|\Lambda\rangle=|0,0\rangle, \\
\left|\Sigma^{-}\right\rangle=|1,-1\rangle ; \quad\left|\Sigma^{0}\right\rangle=|1,0\rangle ; \quad\left|\Sigma^{+}\right\rangle=-|1,+1\rangle, \\
\left|\Xi^{-}\right\rangle=-|1 / 2,-1 / 2\rangle ; \quad\left|\Xi^{0}\right\rangle=|1 / 2,+1 / 2\rangle,
\end{gathered}
$$

it is easy to obtain the Pauli operator in the coupled-isospin basis, $Q_{B \tilde{B}}\left(k, K ; T, M_{T}\right)$, for each strangeness sector. Note that in the following expressions we have only retained the dependence on the isospin labels.

\section{Strangeness 0}

$$
\begin{gathered}
Q_{N N}\left(T=0, M_{T}=0\right)=\frac{1}{2}\left(Q_{p n}+Q_{n p}\right), \\
Q_{N N}\left(T=1, M_{T}=-1\right)=Q_{n n}, \\
Q_{N N}\left(T=1, M_{T}=0\right)=\frac{1}{2}\left(Q_{p n}+Q_{n p}\right), \\
Q_{N N}\left(T=1, M_{T}=+1\right)=Q_{p p} .
\end{gathered}
$$

\section{Strangeness -1}

$$
\begin{aligned}
& Q_{\Lambda N}\left(T=\frac{1}{2}, M_{T}=-\frac{1}{2}\right)=Q_{\Lambda n}, \\
& Q_{\Lambda N}\left(T=\frac{1}{2}, M_{T}=+\frac{1}{2}\right)=Q_{\Lambda p},
\end{aligned}
$$

$$
\begin{aligned}
& Q_{\Sigma N}\left(T=\frac{1}{2}, M_{T}=-\frac{1}{2}\right)=\frac{1}{3} Q_{\Sigma^{0} n}+\frac{2}{3} Q_{\Sigma^{-} p}, \\
& Q_{\Sigma N}\left(T=\frac{1}{2}, M_{T}=+\frac{1}{2}\right)=\frac{2}{3} Q_{\Sigma^{+}{ }_{n}}+\frac{1}{3} Q_{\Sigma^{0} p},
\end{aligned}
$$

$$
\begin{gathered}
Q_{\Sigma N}\left(T=\frac{3}{2}, M_{T}=-\frac{3}{2}\right)=Q_{\Sigma^{-} n}, \\
Q_{\Sigma N}\left(T=\frac{3}{2}, M_{T}=-\frac{1}{2}\right)=\frac{2}{3} Q_{\Sigma^{0} n}+\frac{1}{3} Q_{\Sigma^{-} p}, \\
Q_{\Sigma N}\left(T=\frac{3}{2}, M_{T}=+\frac{1}{2}\right)=\frac{1}{3} Q_{\Sigma^{+} n}+\frac{2}{3} Q_{\Sigma^{0} p},
\end{gathered}
$$




$$
\begin{aligned}
& Q_{\Sigma N}\left(T=\frac{3}{2}, M_{T}=+\frac{3}{2}\right)=Q_{\Sigma+p} \\
& \text { 3. Strangeness }-2 \\
& Q_{\Lambda \Lambda}\left(T=0, M_{T}=0\right)=Q_{\Lambda \Lambda}, \\
& Q_{\Xi N}\left(T=0, M_{T}=0\right)=\frac{1}{2}\left(Q_{\Xi^{-} p}+Q_{\Xi^{0}{ }_{n}}\right), \\
& Q_{\Sigma \Sigma}\left(T=0, M_{T}=0\right)=\frac{1}{3}\left(Q_{\Sigma^{+} \Sigma^{-}}+Q_{\Sigma^{0} \Sigma^{0}}+Q_{\Sigma^{-} \Sigma^{+}}\right), \\
& Q_{\Xi N}\left(T=1, M_{T}=-1\right)=Q_{\Xi^{-} n}, \\
& Q_{\Xi N}\left(T=1, M_{T}=0\right)=\frac{1}{2}\left(Q_{\Xi^{-} p}+Q_{\Xi^{0} n}\right), \\
& Q_{\Xi N}\left(T=1, M_{T}=+1\right)=Q_{\Xi^{0} p}, \\
& Q_{\Lambda \Sigma}\left(T=1, M_{T}=-1\right)=Q_{\Lambda \Sigma^{-}}, \\
& Q_{\Lambda \Sigma}\left(T=1, M_{T}=0\right)=Q_{\Lambda \Sigma^{0}}, \\
& Q_{\Lambda \Sigma}\left(T=1, M_{T}=+1\right)=Q_{\Lambda \Sigma^{+}}, \\
& Q_{\Sigma \Sigma}\left(T=1, M_{T}=-1\right)=\frac{1}{2}\left(Q_{\Sigma^{0} \Sigma^{-}}+Q_{\Sigma^{-} \Sigma^{0}}\right), \\
& Q_{\Sigma \Sigma}\left(T=1, M_{T}=0\right)=\frac{1}{2}\left(Q_{\Sigma^{+} \Sigma^{-}}+Q_{\Sigma^{-} \Sigma^{+}}\right), \\
& Q_{\Sigma \Sigma}\left(T=1, M_{T}=+1\right)=\frac{1}{2}\left(Q_{\Sigma^{0} \Sigma^{+}}+Q_{\Sigma^{+} \Sigma^{0}}\right) \\
& Q_{\Sigma \Sigma}\left(T=2, M_{T}=-2\right)=Q_{\Sigma^{-} \Sigma^{-}}, \\
& Q_{\Sigma \Sigma}\left(T=2, M_{T}=-1\right)=\frac{1}{2}\left(Q_{\Sigma^{0} \Sigma^{-}}+Q_{\Sigma^{-} \Sigma^{0}}\right), \\
& Q_{\Sigma \Sigma}\left(T=2, M_{T}=0\right)=\frac{1}{6} Q_{\Sigma^{+} \Sigma^{-}}+\frac{2}{3} Q_{\Sigma^{0} \Sigma^{0}}+\frac{1}{6} Q_{\Sigma^{-} \Sigma^{+}}, \\
& Q_{\Sigma \Sigma}\left(T=2, M_{T}=+1\right)=\frac{1}{2}\left(Q_{\Sigma^{0} \Sigma^{+}}+Q_{\Sigma^{+} \Sigma^{0}}\right), \\
& Q_{\Sigma \Sigma}\left(T=2, M_{T}=+2\right)=Q_{\Sigma^{+} \Sigma^{+}} . \\
& \text {4. Strangeness }-3 \\
& Q_{\Lambda \Xi}\left(T=\frac{1}{2}, M_{T}=-\frac{1}{2}\right)=Q_{\Lambda \Xi^{-}}, \\
& Q_{\Lambda \Xi}\left(T=\frac{1}{2}, M_{T}=+\frac{1}{2}\right)=Q_{\Lambda \Xi 0},
\end{aligned}
$$

$$
Q_{\Sigma \Xi}\left(T=\frac{1}{2}, M_{T}=-\frac{1}{2}\right)=\frac{1}{3} Q_{\Sigma^{0} \Xi^{-}}+\frac{2}{3} Q_{\Sigma^{-} \Xi^{0}},
$$

$$
\begin{gathered}
Q_{\Sigma \Xi}\left(T=\frac{1}{2}, M_{T}=+\frac{1}{2}\right)=\frac{2}{3} Q_{\Sigma^{+} \Xi^{-}}+\frac{1}{3} Q_{\Sigma^{0} \Xi^{0}}, \\
Q_{\Sigma \Xi}\left(T=\frac{3}{2}, M_{T}=-\frac{3}{2}\right)=Q_{\Sigma^{-} \Xi^{-}}, \\
Q_{\Sigma \Xi}\left(T=\frac{3}{2}, M_{T}=-\frac{1}{2}\right)=\frac{2}{3} Q_{\Sigma^{0} \Xi^{-}}+\frac{1}{3} Q_{\Sigma^{-} \Xi^{0}}, \\
Q_{\Sigma \Xi}\left(T=\frac{3}{2}, M_{T}=+\frac{1}{2}\right)=\frac{1}{3} Q_{\Sigma^{+} \Xi^{-}+\frac{2}{3} Q_{\Sigma^{0} \Xi^{0}},} \\
Q_{\Sigma \Xi}\left(T=\frac{3}{2}, M_{T}=+\frac{3}{2}\right)=Q_{\Sigma^{+} \Xi^{0}} .
\end{gathered}
$$

\section{Strangeness -4}

$$
\begin{gathered}
Q_{\Xi \Xi}\left(T=0, M_{T}=0\right)=\frac{1}{2}\left(Q_{\Xi^{0} \Xi^{-}}+Q_{\Xi^{-} \Xi^{0}}\right), \\
Q_{\Xi \Xi}\left(T=1, M_{T}=-1\right)=Q_{\Xi^{-} \Xi^{-}}, \\
Q_{\Xi \Xi}\left(T=1, M_{T}=0\right)=\frac{1}{2}\left(Q_{\Xi^{0} \Xi^{-}}+Q_{\Xi^{-} \Xi^{0}}\right), \\
Q_{\Xi \Xi}\left(T=1, M_{T}=+1\right)=Q_{\Xi^{0} \Xi^{0}} .
\end{gathered}
$$

From the above expressions it is easy to see that in isospin saturated matter matter (i.e., $k_{F}^{(n)}=k_{F}^{(p)}, \quad k_{F}^{\left(\Sigma^{+}\right)}=k_{F}^{\left(\Sigma^{0}\right)}$ $=k_{F}^{\left(\Sigma^{-}\right)}$and $\left.k_{F}^{\left(\Xi^{0}\right)}=k_{F}^{\left(\Xi^{-}\right)}\right)$the dependence on the third component of isospin disappears.

\section{APPENDIX B: AVERAGE OF THE CENTER-OF-MASS AND HOLE MOMENTA}

In this appendix we show how to compute an appropriate angular average of the center-of-mass momentum of the pair $B_{1} B_{2}$ and the hole momentum $\vec{k}_{B_{2}}$ which enters in the determination of the starting energy in Eqs. (2) and (3). The center-of-mass momentum $\vec{K}$ and the relative momentum $\vec{k}$ of the pair $B_{1} B_{2}$ are defined in the following way:

$$
\begin{gathered}
\vec{K}=\vec{k}_{B_{1}}+\vec{k}_{B_{2}}, \\
\vec{k}=\frac{M_{B_{2}} \vec{k}_{B_{1}}-M_{B_{1}} \vec{k}_{B_{2}}}{M_{B_{1}}+M_{B_{2}}}=\beta \vec{k}_{B_{1}}-\alpha \vec{k}_{B_{2}} .
\end{gathered}
$$


From the above expressions it is easy to write $\vec{K}$ and $\vec{k}_{B_{2}}$ in terms of the extrenal momentum $\vec{k}_{B_{1}}$ and the relative momentum $\vec{k}$, which is used as integration variable in Eqs. (2) and (3):

$$
\begin{aligned}
\vec{K} & =\frac{1}{\alpha}\left(\vec{k}_{B_{1}}-\vec{k}\right), \\
\vec{k}_{B_{2}} & =\frac{1}{\alpha}\left(\beta \vec{k}_{B_{1}}-\vec{k}\right) .
\end{aligned}
$$

The angle average of the center-of-mass momentum is defined as

$$
\overline{K^{2}}\left(k_{B_{1}}, k\right)=\frac{\int d(\cos \theta) K^{2}\left(k_{B_{1}}, k, \cos \theta\right)}{\int d(\cos \theta)},
$$

where $K^{2}\left(k_{B_{1}}, k, \cos \theta\right)=\left(1 / \alpha^{2}\right)\left(k_{B_{1}}^{2}+k^{2}-2 k_{B_{1}} k \cos \theta\right)$, with $\theta$ being the angle between $\vec{k}_{B_{1}}$ and $\vec{k}$. The integration runs over all the angles for which $\left|\vec{k}_{B_{2}}\right|<k_{F}^{\left(B_{2}\right)}$. Similarly, for the hole momentum we have

$$
\overline{k_{B_{2}}^{2}}\left(k_{B_{1}}, k\right)=\frac{\int d(\cos \theta) k_{B_{2}}^{2}\left(k_{B_{1}}, k, \cos \theta\right)}{\int d(\cos \theta)},
$$

where $k_{B_{2}}^{2}\left(k_{B_{1}}, k, \cos \theta\right)=\left(1 / \alpha^{2}\right)\left(\beta^{2} k_{B_{1}}^{2}+k^{2}-2 \beta k_{B_{1}} k \cos \theta\right)$.

We can distinguish two cases in performing the angular integrals, $\beta k_{B_{1}}<\alpha k_{F}^{\left(B_{2}\right)}$ and $\beta k_{B_{1}}>\alpha k_{F}^{\left(B_{2}\right)}$. In the first case, we have two possibilities $0<k<\alpha k_{F}^{\left(B_{2}\right)}-k_{B_{1}}$, for which all angle values are allowed, giving the result

$$
\begin{gathered}
\overline{K^{2}}\left(k_{B_{1}}, k\right)=\frac{1}{\alpha^{2}}\left[k_{B_{1}}^{2}+k^{2}\right], \\
\overline{k_{B_{2}}^{2}}\left(k_{B_{1}}, k\right)=\frac{1}{\alpha^{2}}\left[\beta^{2} k_{B_{1}}^{2}+k^{2}\right],
\end{gathered}
$$

and $\alpha k_{F}^{\left(B_{2}\right)}-\beta k_{B_{1}}<k<\alpha k_{F}^{\left(B_{2}\right)}+\beta k_{B_{1}}$, which have the following upper limit in the value of $\cos \theta$ :

$$
(\cos \theta)_{\max }=\frac{k^{2}+\left(\beta k_{B_{1}}\right)^{2}-\left(\alpha k_{F}^{\left(B_{2}\right)}\right)^{2}}{2 \beta k k_{B_{1}}},
$$

giving the result

$$
\overline{K^{2}}\left(k_{B_{1}}, k\right)=\frac{1}{\alpha^{2}}\left[k_{B_{1}}^{2}+k^{2}-\frac{1}{2 \beta}\left[\left(\beta k_{B_{1}}+k\right)^{2}-\left(\alpha k_{F}^{\left(B_{2}\right)}\right)^{2}\right]\right]
$$

$$
\overline{k_{B_{2}}^{2}}\left(k_{B_{1}}, k\right)=\frac{1}{\alpha^{2}}\left[\beta^{2} k_{B_{1}}^{2}+k^{2}-\frac{1}{2}\left[\left(\beta k_{B_{1}}+k\right)^{2}-\left(\alpha k_{F}^{\left(B_{2}\right)}\right)^{2}\right]\right] .
$$

In the second case, there is only one possibility $\beta k_{B_{1}}$ $-\alpha k_{F}^{\left(B_{2}\right)}<k<\alpha k_{F}^{\left(B_{2}\right)}+\beta k_{B_{1}}$ and the result is the same as in the previous case for the zone $\alpha k_{F}^{\left(B_{2}\right)}-\beta k_{B_{1}}<k<\alpha k_{F}^{\left(B_{2}\right)}$ $+\beta k_{B_{1}}$. The result for the values $0<k<\beta k_{B_{1}}-\alpha k_{F}^{\left(B_{2}\right)}$ is zero because $\vec{k}_{B_{2}}$ is always larger than its Fermi sea.

This kind of average defines an angle-independent centerof-mass momentum and a hole momentum (and therefore a starting energy) for each pair $k_{B_{1}}, k$, so the angular integration in Eqs. (2) and (3) can be performed analytically. Nevertheless, we still require to solve the $G$-matrix equation for each pair of values $k_{B_{1}}$ and $k$, making the calculation very time consuming. In order to speed up the procedure we introduce another average, which gives equivalent results and saves a lot of time. For each external momentum $k_{B_{1}}$, we will only need to solve the $G$-matrix equation for two values of the center-of-mass and hole momenta, which are obtained from

$$
\overline{K^{2}}\left(k_{B_{1}}\right)=\frac{\int d^{3} k K^{2}\left(k_{B_{1}}, k, \cos \theta\right)}{\int d^{3} k},
$$

$$
\overline{k_{B_{2}}^{2}}\left(k_{B_{1}}\right)=\frac{\int d^{3} k k_{B_{2}}^{2}\left(k_{B_{1}}, k, \cos \theta\right)}{\int d^{3} k},
$$

by limiting the integral over the modulus of $\vec{k}$ to the two possibilities mentioned above. As before, we have the same cases $\beta k_{B_{1}}<\alpha k_{F}^{\left(B_{2}\right)}$ and $\beta k_{B_{1}}>\alpha k_{F}^{\left(B_{2}\right)}$. Let us consider the first case. Now, when the integral over $k$ in Eqs. (B12) and (B13) is limited to $0<k<\alpha k_{F}^{\left(B_{2}\right)}-\beta k_{B_{1}}$ we have

$$
\begin{aligned}
& \overline{K^{2}}\left(k_{B_{1}}\right)=\frac{1}{\alpha^{2}}\left[k_{B_{1}}^{2}+\frac{3}{5}\left(\alpha k_{F}^{\left(B_{2}\right)}-\beta k_{B_{1}}\right)^{2}\right], \\
& \overline{k_{B_{2}}^{2}}\left(k_{B_{1}}\right)=\frac{1}{\alpha^{2}}\left[\beta^{2} k_{B_{1}}^{2}+\frac{3}{5}\left(\alpha k_{F}^{\left(B_{2}\right)}-\beta k_{B_{1}}\right)^{2}\right],
\end{aligned}
$$

whereas in the zone $\alpha k_{F}^{\left(B_{2}\right)}-\beta k_{B_{1}}<k<\alpha k_{F}^{\left(B_{2}\right)}+\beta k_{B_{1}}$ the expressions are a little bit more tedious: 


$$
\begin{aligned}
\overline{K^{2}}\left(k_{B_{1}}\right)= & -\frac{\beta^{2}\left(1+\beta^{2}\right)}{\alpha} k_{F}^{\left(B_{2}\right)} k_{B_{1}}^{4}+\beta\left(1+2 \beta^{2}\right) k_{F}^{\left(B_{2}\right) 2} k_{B_{1}}^{3} \\
& +\left(\frac{\alpha^{3}}{2}+\frac{\alpha}{12}\left(4 \beta-26 \beta^{2}-6\right)\right) k_{F}^{\left(B_{2}\right) 3} k_{B_{1}}^{2} \\
& \left.+\alpha^{2} \beta k_{F}^{\left(B_{2}\right) 4} k_{B_{1}}+\frac{\beta^{3}}{15 \alpha^{2}}\left(5+3 \beta^{2}\right) k_{B_{1}}^{5}\right] \\
& \times\left[\left(\alpha k_{F}^{\left(B_{2}\right)}\right)^{2} \beta k_{B_{1}}+\frac{1}{3}\left(\beta k_{B_{1}}\right)^{3}\right. \\
& \left.-\alpha k_{F}^{\left(B_{2}\right)}\left(\beta k_{B_{1}}\right)^{2}\right]^{-1}, \\
\overline{k_{B_{2}}^{2}}\left(k_{B_{1}}\right)= & -\frac{2 \beta^{4}}{\alpha} k_{F}^{\left(B_{2}\right)} k_{B_{1}}^{4}+3 \beta^{3} k_{F}^{\left(B_{2}\right) 2} k_{B_{1}}^{3} \\
& \left.-\frac{7}{3} \alpha \beta^{2} k_{F}^{\left(B_{2}\right) 3} k_{B_{1}}^{2}+\alpha^{2} \beta k_{F}^{\left(B_{2}\right) 4} k_{B_{1}}+\frac{8 \beta^{5}}{15 \alpha^{2}} k_{B_{1}}^{5}\right]
\end{aligned}
$$

[1] N. K. Glendenning, Phys. Rev. D 46, 1274 (1992).

[2] J. Lattimer, C. Pethick, M. Prakash, and P. Haensel, Phys. Rev. Lett. 66, 2701 (1991).

[3] G. B. Cook, S. L. Shapiro, and S. A. Teukolsky, Astrophys. J. 424, 823 (1994).

[4] R. Knorren, M. Prakash, and P. J. Ellis, Phys. Rev. C 52, 3470 (1995).

[5] M. Prakash, I. Bombaci, M. Prakash, P. J. Ellis, J.M. Lattimer, and R. Knorren, Phys. Rep. 280, 1 (1997).

[6] P. J. Ellis, R. Knorren, and M. Prakash, Phys. Lett. B 349, 11 (1995).

[7] J. Schaffner and I. Mishustin, Phys. Rev. C 53, 1416 (1996).

[8] S. Balberg and A. Gal, Nucl. Phys. A625, 435 (1997).

[9] H.-J. Schulze, A. Lejeune, J. Cugnon, M. Baldo, and U. Lombardo, Phys. Lett. B 355, 21 (1995).

[10] H.-J. Schulze, M. Baldo, U. Lombardo, J. Cugnon, and A. Lejeune, Phys. Rev. C 57, 704 (1998).

[11] M. Baldo, G. F. Burgio, and H.-J. Schulze, Phys. Rev. C 58, 3688 (1998).

[12] V. G. J. Stoks and Th. A. Rijken, Phys. Rev. C 59, 3009 (1999).

[13] V. G. J. Stoks and T.-S. H. Lee, Phys. Rev. C 60, 024006

$$
\begin{aligned}
& \times\left[\left(\alpha k_{F}^{\left(B_{2}\right)}\right)^{2} \beta k_{B_{1}}+\frac{1}{3}\left(\beta k_{B_{1}}\right)^{3}\right. \\
& \left.-\alpha k_{F}^{\left(B_{2}\right)}\left(\beta k_{B_{1}}\right)^{2}\right]^{-1} .
\end{aligned}
$$

When $\vec{k}_{B_{1}}=0$ there exists only one zone of integration, 0 $<k<\alpha k_{F}^{\left(B_{2}\right)}$, and the average is very simple:

$$
\overline{K^{2}}\left(k_{B_{1}}\right)=\overline{k_{B_{2}}^{2}}\left(k_{B_{1}}\right)=\frac{3}{5} k_{F}^{\left(B_{2}\right)_{2}} \text {. }
$$

Finally, in the second case $\beta k_{B_{1}}>\alpha k_{F}^{\left(B_{2}\right)}$, there is also only one integration zone $\beta k_{B_{1}}-\alpha k_{F}^{\left(B_{2}\right)}<k<\alpha k_{F}^{\left(B_{2}\right)}+\beta k_{B_{1}}$, and the corresponding averages are

$$
\begin{gathered}
\overline{K^{2}}\left(k_{B_{1}}\right)=\frac{3}{5} k_{F}^{\left(B_{2}\right) 2}+k_{B_{1}}^{2}, \\
\overline{k_{B_{2}}^{2}}\left(k_{B_{1}}\right)=\frac{3}{5} k_{F}^{\left(B_{2}\right) 2} .
\end{gathered}
$$

(1999).

[14] I. Bombaci and U. Lombardo, Phys. Rev. C 44, 1892 (1991).

[15] L. Engvik, M. Hjorth-Jensen, E. Osnes, G. Bao, and E. Østgaard, Phys. Rev. Lett. 73, 2650 (1994).

[16] L. Engvik, M. Hjorth-Jensen, E. Osnes, G. Bao, and E. Фstgaard, Astrophys. J. 469, 794 (1996).

[17] Th. A. Rijken, V. G. J. Stoks, and Y. Yamamoto, Phys. Rev. C 59, 21 (1999).

[18] I. Vidaña, A. Polls, A. Ramos, L. Engvik, and M. HjorthJensen (unpublished).

[19] P. M. M. Maesen, Th. A. Rijken, and J. J. Swart, Phys. Rev. C 40, 2226 (1989).

[20] H. Bandō, T. Motoba, and J. Zofka, Int. J. Mod. Phys. A 5, 4021 (1990).

[21] M. Hjorth-Jensen, A. Polls, A. Ramos, and H. Müther, Nucl. Phys. A605, 458 (1996).

[22] I. Vidaña, A. Polls, A. Ramos, and M. Hjorth-Jensen, Nucl. Phys. A644, 201 (1998).

[23] Y. Yamamoto, T. Motoba, H. Himeno, K. Ikeda, and S. Nagata, Prog. Theor. Phys. Suppl. 117, 36 (1994).

[24] D. J. Millener, C. B. Dover, and A. Gal, Phys. Rev. C 38, 2700 (1988). 